\title{
Nares Occlusion Eliminates Heterosexual Partner Selection without Disrupting Coitus in Ferrets of Both Sexes
}

\author{
Kevin R. Kelliher and Michael J. Baum \\ Department of Biology, Boston University, Boston, Massachusetts 02215
}

Using an airtight $Y$ maze and a new method to induce peripheral anosmia in ferrets, we assessed the contribution of conspecific odors, either alone or in combination with visual and auditory signals, to heterosexual partner preference. Sexually naive ferrets were gonadectomized and treated with sex steroids, after which their nares were either bilaterally occluded using dental impression material or were sham-occluded. Behavioral and histological evidence suggested that nares occlusion blocked access of odors to the main olfactory epithelium for the duration of the study. Sham-occluded females and males preferred to approach odor only or odor plus visual plus auditory cues from opposite-sex conspecifics, whereas naresoccluded ferrets approached opposite- and same-sex cues

The neural mechanisms controlling heterosexual partner choice and coitus are sexually differentiated in mammals (Baum, 1979; reviewed in Adkins-Regan, 1988). In the ferret, a carnivore, the preference of males to approach and copulate with estrous females is organized perinatally via the neural action of testosterone secreted by the testes (Baum et al., 1990a). The expression of male-typical partner preference has been linked to the differentiation in male ferrets of a sexually dimorphic nucleus in the dorsal medial preoptic area/anterior hypothalamus (dmPOA/ AH; for review, see Baum et al., 1990b). Bilateral lesions centered in the dmPOA/AH caused adult male ferrets to prefer to approach and interact with other sexually active male conspecifics instead of with estrous females (Paredes and Baum, 1995). Female ferrets that received similar lesions, like control females, preferred to approach sexually active males. A homosexual partner preference was also seen in males with dmPOA/AH lesions when only distal stimuli (olfactory, visual, and auditory) from stimulus animals were provided (Kindon et al., 1996). Thus neurons in the male's mPOA/AH apparently integrate information about the smell, sound, or physical appearance of conspecifics so that they are motivated to approach a female. By contrast, females, which lack this nucleus, process distal signals from conspecifics so that they seek out a male.

Under seminatural conditions, male and female ferrets use scent marking to maintain separate but overlapping territories

\footnotetext{
Received March 9, 2001; revised May 15, 2001; accepted May 18, 2001.

This research was supported by National Institutes of Health Grant HD21094. We thank the staff of the Boston University Laboratory Animal Care Facility for care of our ferret colony, Robert Kinsland of the Boston University Scientific Instrument Facility for constructing the air-tight Y maze, and Benjamin Sachs for helpful comments on an early draft of this manuscript.

Correspondence should be addressed to Dr. Michael J. Baum, Department of Biology, 5 Cummington Street, Boston University, Boston, MA 02215. E-mail: baum@bio.bu.edu.

Copyright (C) 2001 Society for Neuroscience $\quad 0270-6474 / 01 / 215832-09 \$ 15.00 / 0$
}

equally. All ferrets subsequently mated successfully in tests conducted in a small chamber. When retested in the $Y$ maze, sham-occluded females and males again preferred to approach odor-only or odor plus visual plus auditory cues from oppositesex ferrets, whereas nares-occluded subjects showed no such preference even in tests when a brief physical interaction with tethered stimulus ferrets was allowed after each trial. Our results show that in the ferret, a carnivore, the detection and processing of volatile odors from conspecifics by the main olfactory system is required for heterosexual mate choice.

Key words: anosmia; sexual behavior; sex dimorphism; olfactory bulb; pheromone; c-fos
(Moors and Lavers, 1981; Clapperton et al., 1988). When in breeding condition, both males and females approach and investigate scent deposits from the opposite sex (Chang et al., 2000), suggesting that odors may contribute to heterosexual attraction. The ferret possesses a vomeronasal organ (Weiler et al., 1999) and an associated accessory olfactory bulb (Kelliher et al., 2001). Using Fos protein immunoreactivity (IR) as a marker of neuronal activation, we (Kelliher et al., 1998) found that contact with the soiled bedding of either an estrous female or a stud male activated granule cells in the main olfactory bulb (MOB), but not in the accessory olfactory bulb (AOB) of ferrets of both sexes. This suggests that the main olfactory epithelium (MOE), as opposed to the vomeronasal organ (VNO), is primarily responsible for detecting socially relevant odors in this carnivore. Numerous studies have assessed the relative contribution of the main versus accessory olfactory systems in attracting male mammals to opposite-sex odors and in promoting sexual arousal and coitus (for review, see Doty, 1986); however, very few studies have addressed this issue in females (Edwards and Burge, 1973; Dorries et al., 1997). Also, to our knowledge, no previous studies have directly compared the contribution of olfactory cues to heterosexual partner selection and mating performance in the two sexes. We used an airtight Y maze and a new method (Buchman et al., 1999) for naris occlusion to compare the contributions of volatile odors from conspecifics to sexual partner preference and to mating performance in male and female ferrets before and after they had received coital experience. Finally, the ability of visual and auditory cues as well as direct physical interaction with conspecifics to compensate for anosmia-induced deficits in heterosexual partner selection was assessed.

\section{MATERIALS AND METHODS}

Animals. Prepubertal male and female European ferrets (Mustelo furo), aged 12-16 weeks, were purchased from Marshall Farms (North Rose, NY) and individually housed in modified rabbit cages under a long-day 


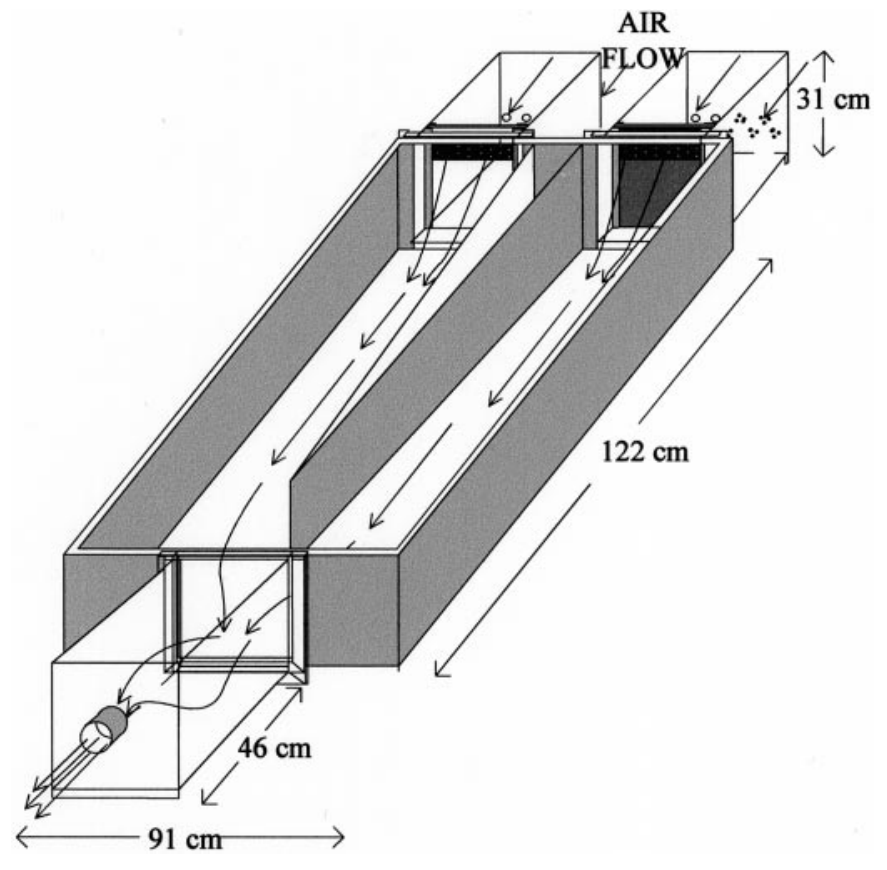

Figure 1. Diagram of the airtight Y maze that was used to assess the effects of bilateral nares occlusion on ferrets' mate recognition and on their capacity to use the peppermint odor as a discriminative stimulus to locate a food reward. Arrows show the direction of air flow through the two goal boxes (right side), into the choice areas, and through the start box (left side) before venting from the room. The doors to the goal boxes were either opaque (for presenting only odor cues) or transparent (for presenting odor plus visual plus auditory cues).

(16/8 hr light/dark cycle) photoperiod. Ferrets were fed moistened Ralston Purina (St. Louis, MO) ferret chow once a day, and water was available ad libitum. Males were castrated, and females were ovohysterectomized under ketamine $(35 \mathrm{mg} / \mathrm{kg})$ and xylazine $(12 \mathrm{mg} / \mathrm{kg})$ anesthesia. Males were subsequently injected daily with testosterone propionate (TP; $5 \mathrm{mg} / \mathrm{kg}$, s.c.) in sesame oil, whereas females received estradiol benzoate $(15 \mu \mathrm{g} / \mathrm{ml}$, s.c.). These treatments elicit the full range of masculine and feminine sexual behaviors in gonadectomized males and females, respectively, and maintain plasma levels of sex steroids that resemble those of gonadally intact male and female ferrets in the breeding condition (for review, see Baum et al., 1990b). Steroid treatments began 4 weeks before the onset of behavioral testing and continued for the entire study. All procedures used in this study were approved by the Boston University Institutional Animal Care and Use Committee.

Y maze apparatus. A Y maze (Fig. 1) was constructed to allow for the independent presentation of odor as opposed to odor plus visual plus auditory cues from conspecifics. The maze was constructed of stainless steel and was covered with airtight Plexiglas panels. An exhaust fan vented out of the room was used to pull air through the goal boxes (which contained stimulus ferrets) and into the choice area and start box of the Y maze. Plexiglas doors of the two goal boxes were either transparent (allowing subjects to see stimulus animals) or opaque. The two goal boxes could be exchanged so that the source of stimulus odors could be easily switched without unwanted odor contamination.

Naris occlusion. The bilateral naris occlusion procedure used (Buchman et al., 1999) was chosen because it induced peripheral anosmia for an indefinite period, thereby allowing a systematic assessment of the ability of visual, auditory, and somatosensory (physical interaction) stimuli as well as coital experience to compensate for the effects of anosmia on heterosexual partner selection. Naris occlusion is well tolerated by ferrets because they, unlike rodents, readily breathe via the mouth. Zinc sulfate irrigation of the olfactory mucosa, which is commonly used to induce peripheral anosmia in rodents, is effective for only 4-5 d because of the continuous replenishment of olfactory receptor neurons (Powers and Winans, 1973). Another method for inducing long-term anosmia is olfactory bulbectomy, which may cause changes in social behavior that are not the result of anosmia per se (Kelly et al., 1997).

After anesthesia (ketamine, $35 \mathrm{mg} / \mathrm{kg}$; and xylazine, $12 \mathrm{mg} / \mathrm{kg}$ ) a topical anesthetic (Cetacaine; Cetylite Industries Inc., Pennsauken, NJ.) was sprayed bilaterally into the nasal cavity $(1 \mathrm{sec}$ spray $=200 \mathrm{mg})$ to suppress the sneezing reflex. Vinylpolysiloxane mix dental impression material (Henry Schein, Port Washington, NY) was injected through Silastic tubing (Dow Corning, Midland, MI) into each nasal cavity. The tubing was inserted to a depth of $8-10 \mathrm{~mm}$, and the impression material was continuously injected while the tubing was slowly withdrawn from of the nasal cavity so that each naris was completely blocked. Shamoccluded animals were treated in the same manner, except that dental impression material was not injected. Subjects were observed closely both during and after recovery from the general anesthesia for signs of respiratory distress. Ferrets tolerated the occlusions well and breathed via their mouths without apparent difficulty. After bilateral naris occlusion, ferrets displayed occasional bouts of sneezing in an apparent attempt to clear their nasal passages. This sneezing usually occurred in the morning around the time animals were fed; sneezing rarely occurred during behavioral testing sessions.

Behavioral confirmation of anosmia after nares occlusion. Before surgery, all subjects were trained to use a peppermint odor as a discriminative stimulus to identify the location of the goal box in the Y maze that contained a food reward. Ferrets were food-deprived by being allowed to eat for only $45 \mathrm{~min}$ immediately after the daily Y maze test session. All animals received four preoperative test sessions followed by two postoperative sessions beginning $5 \mathrm{~d}$ after surgery. On each trial, subjects could choose to approach a goal box that was either empty or contained a glass bowl with $1 \mathrm{gm}$ of Nutri-Cal (EVSCO Pharmaceuticals, Buena, NJ) that was paired with the peppermint odor. The peppermint odor was presented by placing $1 \mathrm{ml}$ of pure peppermint extract (McCormick \& Co., Hunt Valley, MD) onto two gauze pads that were taped to the air inlet and outlet vents of the goal box. Daily test sessions began with ferrets being given $5 \mathrm{~min}$ to explore the Y maze. Then they received 10 "free" trials interspersed with 9 "guided" trials. During free trials, a subject was placed in the start box for $1 \mathrm{~min}$, after which the door was raised so that the subject could approach either the left or the right goal box. The choice and latency to approach were recorded, after which the door of the chosen goal box was opened and the subject was allowed to enter and eat the Nutri-Cal. The subject was then returned to the start box, after which a guided trial was run. Guided trials were identical to the free trials, except that the path to the goal box chosen on the previous free trial was blocked, thereby forcing the subject to approach the other goal box. We thought that nares-occluded, potentially anosmic ferrets would quickly stop performing in the $\mathrm{Y}$ maze if they did not receive food rewards frequently. Therefore, guided trials were interspersed with free trials to provide all subjects with nonolfactory cues to identify the location of the goal box containing the food reward. During each daily test session, the location of the goal box in which the food reward was paired with the peppermint odor was switched three times (e.g., before free trials 2,5 , and 8 ). The subjects' ability to use the peppermint odor to identify the location of the baited goal box in the three free trials that immediately followed each of these location switches was recorded. For each group of ferrets, the mean percentage of correct choices during these postswitch trials was subsequently computed for each of the six test sessions. Data for male and female subjects were analyzed separately using a two-way ANOVA (two independent groups by six repeated measures).

Partner preference tests. Partner preference tests were performed after ferrets had completed the postoperative assessment of olfactory function using the food-motivated peppermint odor discrimination task. All ferrets were sexually naive when they were initially tested in the Y maze for their preference to approach a goal box containing either a stud male or an estrous female. During the first three test sessions, only volatile odors from the stimulus ferrets were presented. This was accomplished by placing an anesthetized male or female ferret, both of which were gonadally intact and in breeding condition, into the respective goal boxes behind an opaque door. Adult male ferrets are approximately twice as large as females; thus it is possible that ferrets normally use visual cues to identify opposite-sex partners. In addition, male and female ferrets make audible clucking sounds that could convey information about gender. Therefore, during the next three test sessions, subjects were presented with volatile odors and the sight and sounds of the stimulus ferrets. An awake male or female ferret was placed in each of the goal boxes behind transparent Plexiglas doors. After the completion of this second series of tests, all subjects were tested for coital behavior in a small compartment (details below). Then all subjects were tested again (three test sessions per stimulus condition) in the $\mathrm{Y}$ maze for their 
preference to approach odors only followed by odor plus visual plus auditory cues from male versus female conspecifics. In a final series of tests, subjects were allowed to choose between approaching and interacting physically for $30 \mathrm{sec}$ after each trial with an estrous female or a stud male that was tethered in the respective goal boxes. The locations of the goal boxes containing the male versus female stimuli were randomized for each training session.

All Y maze tests of partner preference were performed using rules similar to those described above for tests of subjects' ability to use the peppermint odor to locate food. Daily testing sessions included eight free and seven guided trials. Each subject's stimulus choice (male vs female) and approach latency were recorded for each trial. Mean values based on a total of 24 free trials given over three daily test sessions were computed for each stimulus condition. The locations of the goal boxes containing male and female stimulus animals were alternated on successive test days to avoid the possible influence of any directional bias on ferrets' goal box choices. More frequent switching of the location of male and female stimuli in the Y maze goal boxes was avoided, because we sought to assess partner preferences under circumstances when subjects were maximally aware of the location of the alternative stimuli. The entire Y maze was washed with soap and water and wiped with $70 \%$ ethanol between test sessions for each subject.

Individual trials were terminated if a subject made no choice after 180 sec. During the initial test sessions given when the subjects were sexually naive, trials in which no choice was made were repeated immediately, insofar as this occurred infrequently. In tests given after ferrets had received coital experience, the percentage of free trials during which naris-occluded male subjects failed to make a choice increased notably. Therefore, in the later sessions, if a subject did not make a choice, it was assigned an approach latency of $180 \mathrm{sec}$ and recorded as "no choice" for that trial. When computing the percentage of free trials with approaches to the goal boxes containing male versus female cues, only trials in which a choice was made within $180 \mathrm{sec}$ were included. The approach latency scores for each subject were computed on the basis of all 24 free trials given under each stimulus condition. Data were analyzed separately for males and females using two-way ANOVAs (two independent groups by two repeated measures for subjects tested for Y maze preferences before and after coital experience). One nares-occluded female died after completing the initial series (sexually naive condition) of $\mathrm{Y}$ maze preference tests. To conduct repeated measures ANOVAs on preference and latency data, the SigmaStat (SPSS Inc., Chicago, IL) software normally distributed random number generator was used to designate missing values (Winer et al., 1991) for this single female (within 1 SD of each group mean) in the sexually experienced odor-only and odor plus visual plus auditory conditions. Two-tailed $t$ tests were used for between-group comparisons of results collected during trials when physical interaction was allowed with stimulus ferrets.

Tests of coital behavior. After completing the initial series of Y maze tests of sex partner preference, all subjects were tested in a small chamber $\left(61 \mathrm{~cm}^{2}\right)$ on 3 consecutive days for coital behavior shown with an opposite-sex stimulus ferret in breeding condition. Mating in ferrets generally begins with mutual anogenital investigation, after which the male neck grips and mounts the female before exhibiting bouts of pelvic thrusting, which lead to a single, prolonged intromission that is accompanied by ejaculation. A sexually receptive female ferret shows a limp, unresisting posture (accompanied by tail deviation) in response to a male's neck grip and mount, whereas a sexually unreceptive female vigorously resists any attempted neck grip. The latency, frequency, and duration of various coital behaviors, including anogenital investigation, neck gripping, mounting, thrusting, and intromission were scored using a hand-held computer and The Observer software (Noldus Information Technology, Wageningen, The Netherlands). Subjects that failed to achieve (males) or receive (females) two intromissions during the first three test sessions were given additional tests (unscored) until this criterion of coital performance was met, followed by another series of nine Y maze tests of sex partner preference. Then in conjunction with another study (Bakker et al., 2001) concerning the role of social odors in the mating-induced activation of hypothalamic gonadotropin-releasing hormone neurons, female subjects began receiving two daily injections (morning and evening) of estradiol in sesame oil $(15 \mu \mathrm{g} / \mathrm{kg}$, s.c.), whereas males continued to receive TP. After $10 \mathrm{~d}$, all subjects were again placed in a small chamber and allowed to mate until an intromission was achieved (males) or received (females). Behavioral tests were terminated $90 \mathrm{~min}$ after the onset of this intromission, after which subjects were killed by an overdose of sodium pentobarbital, and the brains were removed for histological examination (details below). Coital behaviors were analyzed separately for male and female subjects using two-way ANOVAs (two independent groups by two repeated measures).

Experimental time line. The entire experiment took $72-75$ d. Beginning 3 weeks after gonadectomy and the onset of sex steroid treatments, subjects received peppermint-food reward training in the $\mathrm{Y}$ maze for $6-10 \mathrm{~d}$. Surgery was then performed over a $2 \mathrm{~d}$ period, and $7 \mathrm{~d}$ later the final peppermint-food reward training sessions were conducted over an additional $2 \mathrm{~d}$. Subjects then received Y maze tests of sex partner preference over a $12-15 \mathrm{~d}$ period. Then an initial series of coital behavior tests was conducted over $9-12 \mathrm{~d}$, followed by a second series of Y maze tests of partner preference conducted over a 18-22 d period. The final test of coital behavior was given $2-5 \mathrm{~d}$ later.

Histological assessment of olfactory function. The presence of neuronal Fos-IR in two brain regions that receive olfactory inputs from the MOB, the anterior-cortical amygdala (CoA) and the piriform cortex (Pir), was taken as an index of olfactory function in the different groups of ferrets, all of which were killed after they achieved (males) or received (females) an intromission during their final behavioral test. Fos-IR cells were counted in single brain sections containing the CoA and Pir from both hemispheres. We also compared neuronal Fos-IR in single sections that included the granule cell layer of the left MOB and the cell layer of the left AOB of nares- and sham-occluded animals. These latter regions corresponded to the most caudal MOB site and the AOB region in which Fos-IR cells were counted in male and female ferrets that were placed on soiled estrous female or male bedding in our previous study (Kelliher et al., 1998). Mitral and granule cells are intermingled in the ferret's AOB (Kelliher et al., 2001) and were not distinguishable in sections that were processed for Fos-IR in the present study.

Subjects were killed by an overdose $(100 \mathrm{mg} / \mathrm{kg})$ of sodium pentobarbital and were perfused via the aorta with $50 \mathrm{ml}$ of $0.1 \mathrm{M} \mathrm{PBS,} \mathrm{pH} \mathrm{7.4,}$ followed by $800 \mathrm{ml}$ (for females) or $1000 \mathrm{ml}$ (for males) of $4 \%$ paraformaldehyde in $0.1 \mathrm{M}$ PBS. The brains were removed and post-fixed for 2 $\mathrm{hr}$ in $4 \%$ paraformaldehyde before being cryoprotected in $30 \%$ sucrose and PBS at $4^{\circ} \mathrm{C}$ until they sank. The left olfactory bulb and both hemispheres of the forebrain were cut in the coronal plane at $30 \mu \mathrm{m}$ using a Reichert-Jung SM2000R tabletop sliding microtome. Freefloating sections from sham- and naris-occluded females were processed together, and sections from males in the two treatment groups were later processed together for Fos-IR as described previously (Wersinger and Baum, 1997; Kelliher et al., 1998). Briefly, tissue sections were preincubated in $3 \%$ normal goat serum and $1 \% \mathrm{H}_{2} \mathrm{O}_{2}$ and PBS for $30 \mathrm{~min}$ and then placed in primary Fos antibody (antibody 52 at a dilution of 1:5000; Santa Cruz Biotechnology, Santa Cruz, CA) overnight for $\sim 16 \mathrm{hr}$. Sections were washed four times for $10 \mathrm{~min}$ between each incubation and then incubated for $2 \mathrm{hr}$ in secondary antibody (goat anti-rabbit 1:200; Vector Laboratories, Burlingame, CA). Sections were then incubated for $2 \mathrm{hr}$ in $\mathrm{ABC}$ (1:100; Vector Laboratories) and reacted for $7 \mathrm{~min}$ with nickel DAB (Vector Laboratories). Sections were mounted on gelatincoated slides and coverslipped using Permount.

Slides were coded before analysis so that the experimenter had no knowledge of subjects' sex or treatment. Brain sections were viewed at $500 \times$, and Fos-IR cells present in standard areas of $0.1 \mathrm{~mm}^{2}$ were traced onto paper using a camera lucida microscope attachment. Neuronal Fos data for the Pir and CoA were analyzed separately for each sex using a two-way ANOVA (two independent groups by brain hemisphere). MOB and AOB data were analyzed separately for each sex using two-tailed $t$ tests.

Dissection of the snouts of each naris-occluded subject showed that the dental impression material invariably filled the anterior portion of the nasal cavity. In ferrets, the nasopalatine canal in the roof of the mouth provides access to the VNO (Kelliher et al., 2001). In nares-occluded ferrets, the dental impression material typically extended caudally in the nasal cavity so that it began to fill the nasopalatine canal without entering the vomeronasal duct.

\section{RESULTS \\ Partner preference tests}

\section{Females}

Before coital experience, nares-occluded females approached the male goal box under the odor-only condition on significantly fewer trials than sham-occluded females (Fig. 2, top graph). These group differences persisted when visual and auditory stimuli from 

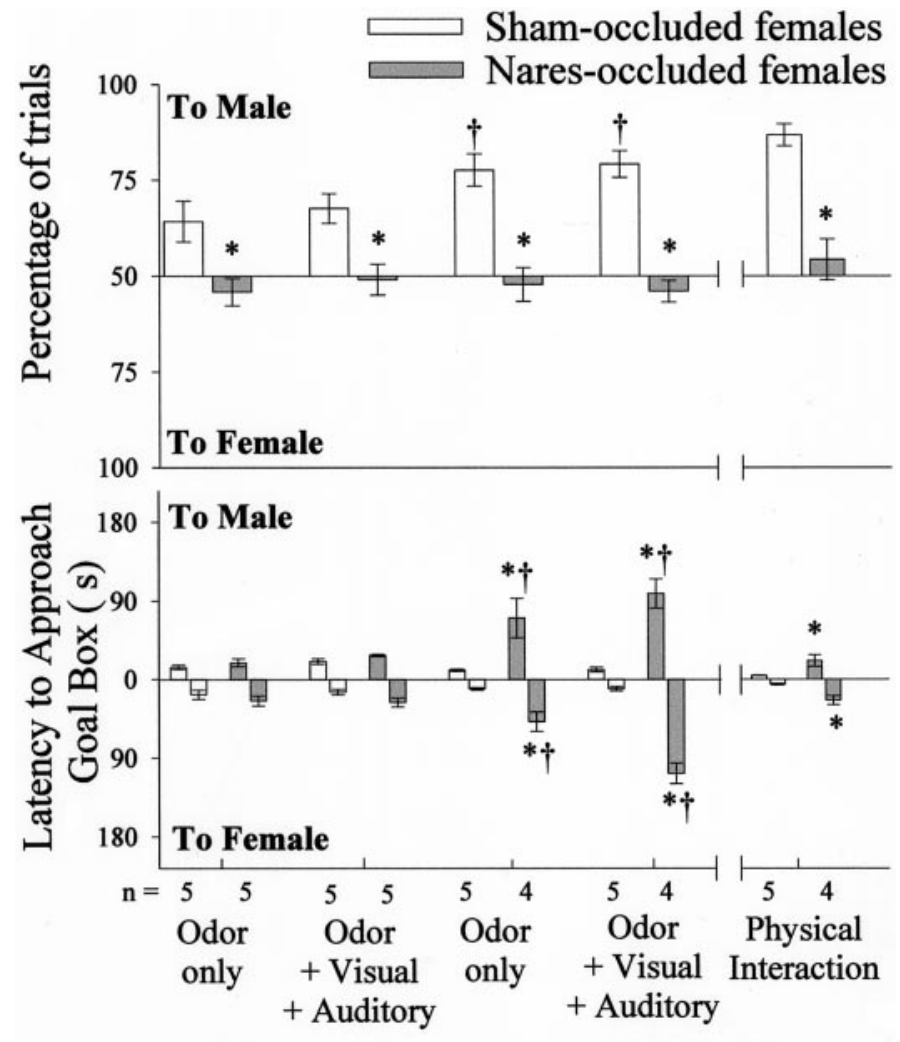

Sexually Naive $\overline{\text { Sexually Experienced }}$

Figure 2. Percentage of free trials (top graph) and approach latencies (bottom graph) in Y maze tests during which ovohysterectomized, estradiol benzoate-treated female ferrets approached a goal box, which provided stimuli from either a sexually active stud male (to male) or an estrous female (to female). Subjects were given $\mathrm{Y}$ maze tests before and again after they had received coital experience. ${ }^{*} p<0.05$, comparisons with sham-occluded females; $t p<0.01$, within-groups comparisons by post hoc Student-Newman-Keuls tests with sexually naive values obtained under the same stimulus conditions. Data are expressed as mean \pm SEM.

male and female stimulus animals were provided in addition to odor cues. The preference of sham-occluded females to approach the male stimuli (both odor-only and odor plus visual plus auditory cues) was significantly enhanced in tests given after they had received coital experience. These sham-occluded control females showed their strongest heterosexual partner preference when allowed to interact physically with the stimulus ferrets during the final series of Y maze trials. No such effect of coital experience was seen in naris-occluded females. Again, they approached the male goal box on significantly fewer trials than sham-occluded females under the odor-only, odor plus visual plus auditory, and physical interaction conditions. Statistical analyses of the percentage of trials to approach the male stimulus under the odor-only condition revealed a significant overall effect of nares occlusion $\left(F_{(1,19)}=21.876 ; p<0.01\right)$ and of coital experience $\left(F_{(1,19)}=\right.$ $6.707 ; p<0.05)$. Under the odor plus visual plus auditory condition, there was a significant occlusion by coital experience interaction $\left(F_{(1,19)}=5.690 ; p<0.05\right)$. In the final series of tests, when physical interaction was allowed after each trial, the stimulus male was approached on significantly fewer trials by naresoccluded than by sham-occluded females (two-tailed $t_{(1,9)}=$ 40.660; $p<0.01)$.
Before coital experience, sham- and nares-occluded females showed similar, relatively short latencies to approach the goal boxes containing a stimulus male and female under both the odor-only and odor plus visual plus auditory cue conditions (Fig. 2 , bottom graph). After females had received coital experience and were again provided either odor-only or odor plus visual plus auditory cues from the stimulus animals, approach latencies to both the male and female goal boxes were significantly greater in naris-occluded than in sham-occluded females. These group differences persisted when subjects were given physical access to the stimulus ferrets after each trial; however, for nares-occluded females the approach latencies were significantly shorter than in previous trials when only distal cues (odor only or odor plus visual plus auditory) were available. In the odor-only condition, there was a statistically significant occlusion by coital experience interaction effect on latency to approach the stimulus male $\left(F_{(1,18)}\right.$ $=26.051 ; p<0.01)$ and the stimulus female $\left(F_{(1,18)}=38.685 ; p<\right.$ $0.01)$. Likewise, in the odor plus visual plus auditory condition, there was a similar significant interaction effect on the latency to approach the stimulus male $\left(F_{(1,18)}=7.799 ; p<0.05\right)$ and the stimulus female $\left(F_{(1,18)}=16.919 ; p<0.01\right)$. These significant interaction effects reflected the longer approach latencies to both goal boxes seen in naris-occluded females but not in shamoccluded controls in tests given after subjects had received coital experience. When allowed physical contact, sham-occluded females had significantly shorter latencies to approach either the male stimulus (two-tailed $t_{(1,8)}=3.092 ; p<0.05$ ) or the female stimulus $\left(t_{(1,8)}=4.828 ; p<0.01\right)$ compared with nares-occluded females. Nares-occluded females had significantly reduced latencies to approach either stimulus when allowed physical interaction as opposed to only odor plus visual plus auditory cues (two-tailed $t_{(1,8)}=3.486 ; p<0.05$ to approach the male; and $t_{(1,8)}=3.092$; $p<0.05$ to approach the female stimulus).

\section{Males}

Before coital experience, nares-occluded males approached the female goal box under the odor-only condition on significantly fewer trials than sham-occluded males (Fig. 3, top graph). Surprisingly, under the odor plus visual plus auditory cue condition, males in the both groups approached the two goal boxes on an equivalent percentage of trials, perhaps because visual and auditory cues stimulated aggression in sham-occluded male subjects toward the stimulus male that competed with their sexual motivation to approach the stimulus female. After they had received coital experience, nares-occluded males continued to approach the female goal box on significantly fewer trials than shamoccluded males under the odor-only, odor plus visual plus auditory, and physical interaction stimulus conditions. Statistical analyses showed that there was a significant overall effect of nares occlusion under both the odor-only $\left(F_{(1,19)}=26.71 ; p<0.01\right)$ and the odor plus visual plus auditory $\left(F_{(1,19)}=7.13 ; p<0.05\right)$ cue conditions. When physical interaction was allowed after each trial, the stimulus female was approached on significantly fewer trials by nares-occluded than by sham-occluded males (two-tailed $\left.t_{(1,9)}=2.44 ; p<0.05\right)$.

Before coital experience, sham- and nares-occluded males showed similar, relatively short latencies to approach the two goal boxes under both the odor-only and odor plus visual plus auditory cue conditions (Fig. 3, bottom graph). After coital experience, approach latencies to both goal boxes were significantly greater in nares-occluded than in sham-occluded males under all three stimulus conditions. Under the odor-only condition, there was a 


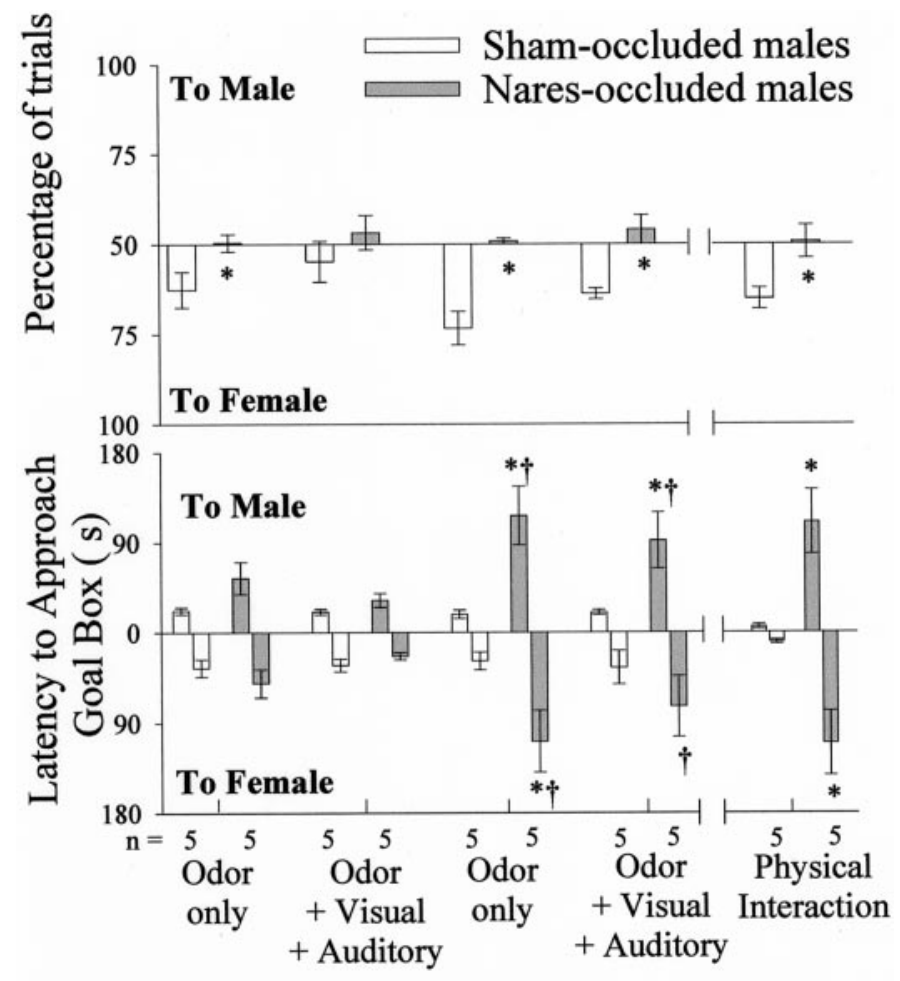

Sexually Naive $\overline{\text { Sexually Experienced }}$

Figure 3. Percentage of free trials (top graph) and approach latencies (bottom graph) in Y maze tests during which castrated, testosterone propionate-treated male ferrets approached a goal box, which provided stimuli from either a sexually active stud male (to male) or an estrous female (to female). Subjects received Y maze tests before and again after they had received coital experience. * $p<0.05$, comparisons with shamoccluded males; $\dagger p<0.01$, within-groups comparisons by post hoc Student-Newman-Keuls tests with sexually naive values obtained under the same stimulus conditions. Data are expressed as mean \pm SEM.

statistically significant occlusion by coital experience interaction effect on latency to approach the stimulus male $\left(F_{(1,18)}=5.541\right.$; $p<0.05)$ and female $\left(F_{(1,18)}=9.684 ; p<0.05\right)$. Likewise, under the odor plus visual plus auditory condition, there was a similar significant interaction effect on the latency to approach the stimulus male $\left(F_{(1,18)}=5.608 ; p<0.05\right)$. These significant interaction effects reflected the longer approach latencies to one or the other goal box seen in nares-occluded males but not in sham-occluded controls in tests given after subjects had received coital experience. When allowed physical interaction after each trial, naresoccluded males had significantly longer latencies than shamoccluded males to approach either the male (two-tailed $t_{(1,8)}=$ $3.962 ; p<0.01)$ or the female $\left(t_{(1,8)}=3.782 ; p<0.01\right)$ stimulus ferrets.

\section{Coital performance}

\section{Females}

In initial tests with a stud male, there were no significant differences between nares- and sham-occluded females (Table 1). Nares-occluded females spent significantly less time than shamoccluded females displaying anogenital investigation toward their male partners $\left(t_{(1,8)}=186.667 ; p<0.01\right)$. When tested again with a stud male at the end of the study, there were again no quantitative differences in sexual behaviors displayed by nares- and sham-occluded females, all of which received an intromission.

\section{Males}

In initial tests of coital performance, the latency to neck grip the stimulus female was significantly shorter $\left(t_{(1,8)}=2.439 ; p<0.05\right)$ in nares-occluded than in sham-occluded males (Table 1). This correlates with the observation that nares-occluded males spent significantly less time $\left(t_{(1,8)}=6.058 ; p<0.05\right)$ than shamoccluded males displaying anogenital investigation toward stimulus females. Other parameters of masculine coital performance, including the occurrence of intromission, were similar in the two groups of males in these initial tests as well as in the test given at the end of the study.

\section{Independent behavioral evidence of anosmia after nares occlusion}

Over the four preoperative test sessions, all subjects acquired the ability to use the peppermint odor to locate the food reward during a high percentage of free Y maze trials given immediately after a switch in the location of the baited goal box (Fig. 4). After sham occlusion, both female and male ferrets continued to identify correctly the location of food using the peppermint odor, whereas the percentage of correct choices made by the naresoccluded males and females dropped significantly. During the postoperative test sessions, nares-occluded subjects resumed their approaches to the food reward once they had located it during the guided trial that was given immediately after the free trial that followed the switch in the location of the baited goal box (data not shown). The existence of a statistically significant occlusion by repeated measures interaction effect for female $\left(F_{(5,59)}=3.098\right.$; $p<0.05)$ and male $\left(F_{(5,59)}=4.190 ; p<0.01\right)$ subjects suggests that a significant degree of generalized postoperative anosmia was induced by bilateral naris occlusion in ferrets of each sex. If nares-occluded subjects had been completely unable to detect the peppermint odor that was paired with a food reward in these tests, they should have approached the baited goal box on zero of three $(0 \%)$ free trials that followed the three bait location switches performed during each test session. The fact that naresoccluded females and males averaged $\sim 30 \%$ correct choices (contrasted with 80 and $90 \%$ correct choices by sham-occluded females and males, respectively) during these particular free trials raises the possibility that nares-occluded subjects retained a residual ability to smell peppermint. Still, the significant differences between nares- and sham-occluded ferrets on performance in this food-motivated olfactory discrimination task, coupled with the parallel group differences in female and male ferrets' preference to approach odors only of an opposite-sex stimulus animal (Figs. 2 and 3), suggest that a significant degree of anosmia was induced in both sexes by bilateral naris occlusion.

\section{Histological evidence of anosmia after naris occlusion}

Mitral and tufted cells in the MOB project directly to the CoA and Pir, among other regions. Thus additional evidence of olfactory dysfunction among nares-occluded ferrets stems from the finding (Fig. 5) that these subjects had significantly fewer Fos-IR cells in these two regions when they were killed after the final test of mating behavior. Statistical analysis showed that there were significant effects of nares occlusion on the number of Fos-IR cells in the Pir of females $\left(F_{(1,17)}=54.384 ; p<0.001\right)$ and males $\left(F_{(1,17)}=23.993 ; p<0.001\right)$ and in the CoA of females $\left(F_{(1,17)}=\right.$ $120.408 ; p<0.001)$ and males $\left(F_{(1,17)}=19.349 ; p<0.001\right)$. Equivalent reductions in the numbers of Fos-IR cells were seen in the two hemispheres of nares-occluded subjects, suggesting that 


\begin{tabular}{|c|c|c|c|c|c|c|c|c|}
\hline Group & $n$ & $\begin{array}{l}\text { Neck grip }^{a} \\
\text { (latency, sec) }\end{array}$ & $\begin{array}{l}\text { Mount }^{a} \\
\text { (latency, sec) }\end{array}$ & $\begin{array}{l}\text { Thrusting }^{a} \\
\text { (latency, sec) }\end{array}$ & $\begin{array}{l}\text { Intromission }^{a} \\
\text { (latency, sec) }^{2}\end{array}$ & Intromission $^{b}$ & $\begin{array}{l}\text { Intromission }^{a} \\
\text { (duration, sec) }\end{array}$ & $\begin{array}{l}\text { AGI } \\
\text { (duration, sec) }\end{array}$ \\
\hline \multicolumn{9}{|l|}{ Females } \\
\hline \multicolumn{9}{|l|}{ Initial test } \\
\hline Sham-occluded & 5 & $80 \pm 20$ & $120 \pm 25$ & $213 \pm 17$ & $1006 \pm 61$ & $5 / 5$ & $918 \pm 118$ & $13 \pm 1$ \\
\hline Nares-occluded & 4 & $85 \pm 9$ & $114 \pm 12$ & $260 \pm 25$ & $1408 \pm 239$ & $2 / 4$ & $940 \pm 133$ & $1 \pm 0^{*}$ \\
\hline \multicolumn{9}{|l|}{ End of study } \\
\hline Sham-occluded & 5 & $47 \pm 6$ & $103 \pm 33$ & $388 \pm 104$ & $881 \pm 249$ & $5 / 5$ & $\mathrm{NC}$ & $\mathrm{NC}$ \\
\hline Nares-occluded & 4 & $146 \pm 60$ & $215 \pm 94$ & $426 \pm 61$ & $1102 \pm 317$ & $4 / 4$ & $\mathrm{NC}$ & $\mathrm{NC}$ \\
\hline \multicolumn{9}{|l|}{ Males } \\
\hline \multicolumn{9}{|l|}{ Initial test } \\
\hline Sham-occluded & 5 & $108 \pm 23$ & $116 \pm 45$ & $606 \pm 167$ & $1558 \pm 146$ & $3 / 5$ & $604 \pm 180$ & $38 \pm 15$ \\
\hline Nares-occluded & 5 & $45 \pm 12^{*}$ & $64 \pm 14$ & $258 \pm 72$ & $1470 \pm 94$ & $3 / 5$ & $826 \pm 97$ & $1 \pm 1^{*}$ \\
\hline \multicolumn{9}{|l|}{ End of Study } \\
\hline Sham-occluded & 5 & $102 \pm 12$ & $169 \pm 35$ & $302 \pm 42$ & $993 \pm 214$ & $5 / 5$ & $4267 \pm 289$ & $\mathrm{NC}$ \\
\hline Nares-occluded & 5 & $43 \pm 30$ & $69 \pm 44$ & $308 \pm 167$ & $893 \pm 234$ & $5 / 5$ & $3122 \pm 859$ & $\mathrm{NC}$ \\
\hline
\end{tabular}

Data are expressed as mean \pm SEM. AGI, Anogenital investigation; NC, data not collected.

${ }^{a}$ By definition, behaviors listed were received by female and performed by male subjects.

${ }^{b}$ Proportion of ferrets receiving or achieving at least one intromission.

${ }^{*} p<0.01$; Student-Neuman-Keuls post hoc comparisons with same-sex sham-occluded subjects.

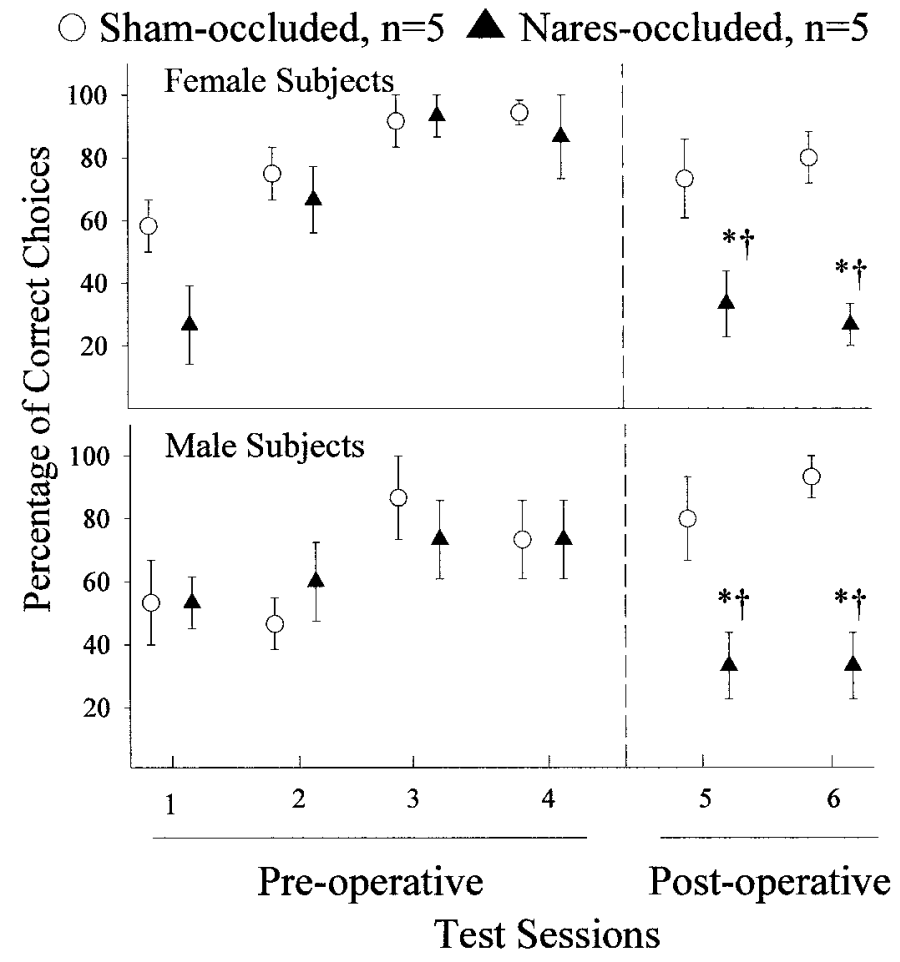

Figure 4. Percentage of trials in which food-deprived female (top graph) and male (bottom graph) ferrets successfully used the peppermint odor as a discriminative stimulus to identify the Y maze goal box that contained the Nutri-Cal food reward. Groups of ferrets were tested both before (Pre-operative) and after (Post-operative) their nares were bilaterally occluded or sham-occluded. ${ }^{*} p<0.05$, comparisons with sham-occluded values in the same postoperative behavioral test session; $\uparrow p<0.01$, within-groups comparisons with values from preoperative test 4 by post hoc Student-Newman-Keuls tests. Data are expressed as mean \pm SEM.

the dental impression material successfully occluded both nares in all subjects.

In the MOB site chosen for analysis, nares-occluded males and females had significantly fewer Fos-IR granule cells than sham- occluded subjects for both females $\left(t_{(1,7)}=5.960 ; p<0.01\right)$ and males $\left(t_{(1,8)}=6.367 ; p<0.01\right.$; Fig. 6$)$. No such group differences were seen in the cell layer of the AOB.

\section{DISCUSSION}

Both male and female ferrets apparently require volatile olfactory cues from opposite-sex conspecifics to identify and seek them out as mating partners. The ferret is a carnivore with a welldeveloped visual system, and adult males are typically twice as large as females. It is therefore surprising that nares-occluded ferrets failed to make heterosexual partner choices in $\mathrm{Y}$ maze tests when visual cues were provided in addition to odors. Indeed, female sheep can use facial cues to distinguish between male and female conspecifics (Kendrick et al., 1995). Even after receiving coital experience, nares-occluded ferrets continued showing no preference to approach opposite-sex conspecifics when they were tethered in the goal boxes and available for physical interaction after each Y maze trial. Apparently sex-specific odor cues are required for ferrets of both sexes to identify potential mates at a distance. However, when confined with a heterosexual partner, nares-occluded ferrets showed the full range of sexual behaviors, suggesting that odors contribute little to mating per se.

Nares occlusion dramatically reduced neuronal Fos-IR in the MOB but not in the AOB of both male and female ferrets. These results together with the present behavioral findings are consistent with our previous suggestion (Wersinger and Baum, 1997; Kelliher et al., 1998, 2001) that the main as opposed to the accessory olfactory system mediates olfactory communication in this carnivore. The reduction in the levels of Fos-IR in the CoA and Pir of nares-occluded ferrets further suggests that the ability of the MOE to detect odors was significantly reduced for the duration of this study (72-75 d). We cannot, however, completely rule out the possibility that some disruption of VNO-AOB function also occurred in nares-occluded subjects, which contributed to the observed disruption of heterosexual partner preference in these subjects. The ferret possesses a nasopalatine canal via which odor stimuli from either the mouth or the nasal cavity potentially gain access to the VNO (Kelliher et al., 2001). Postmortem dissection of nares-occluded ferrets showed that dental impres- 

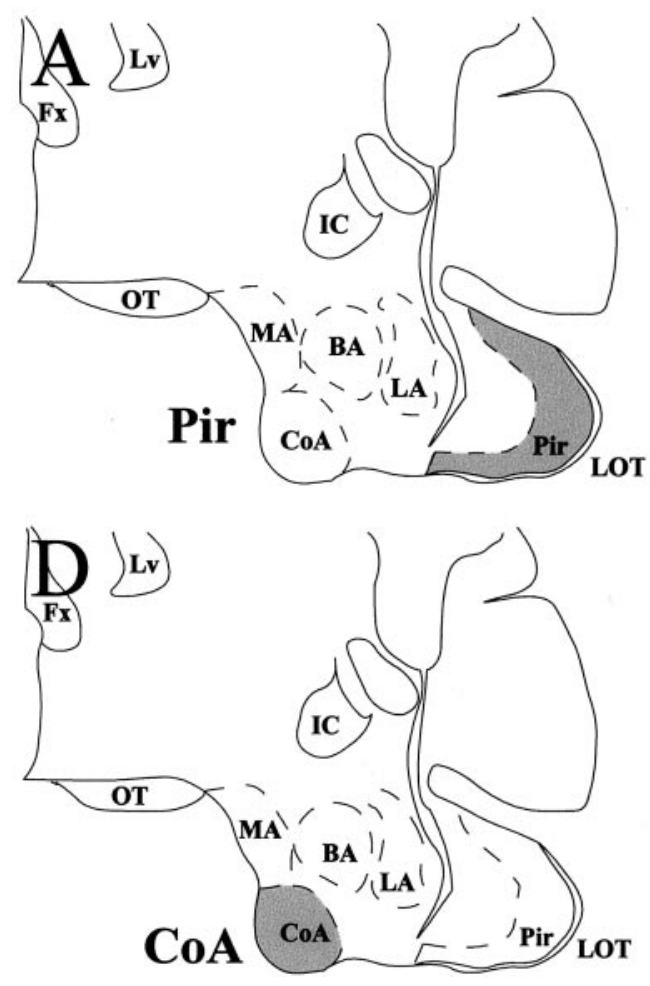
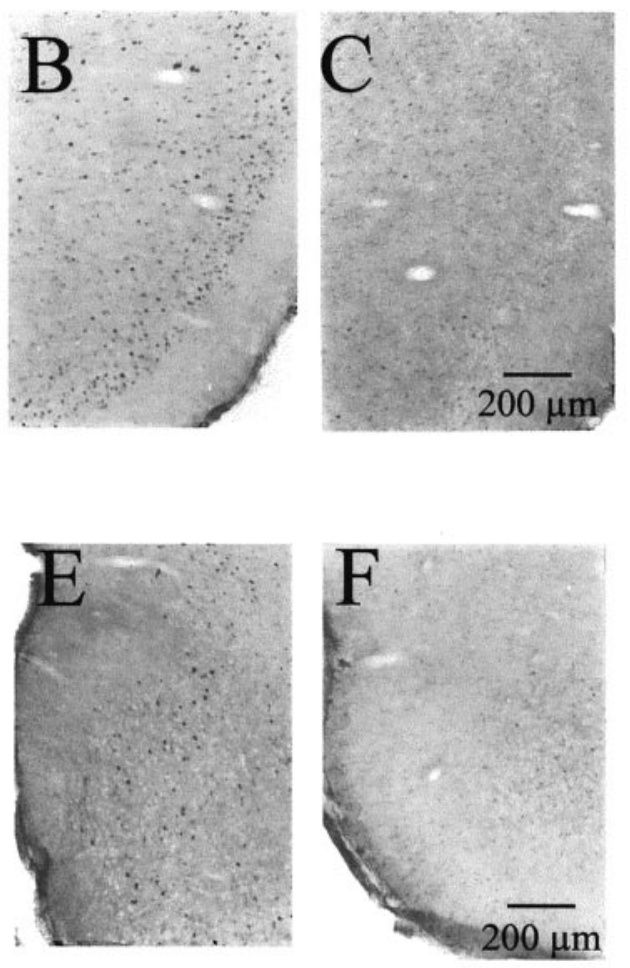

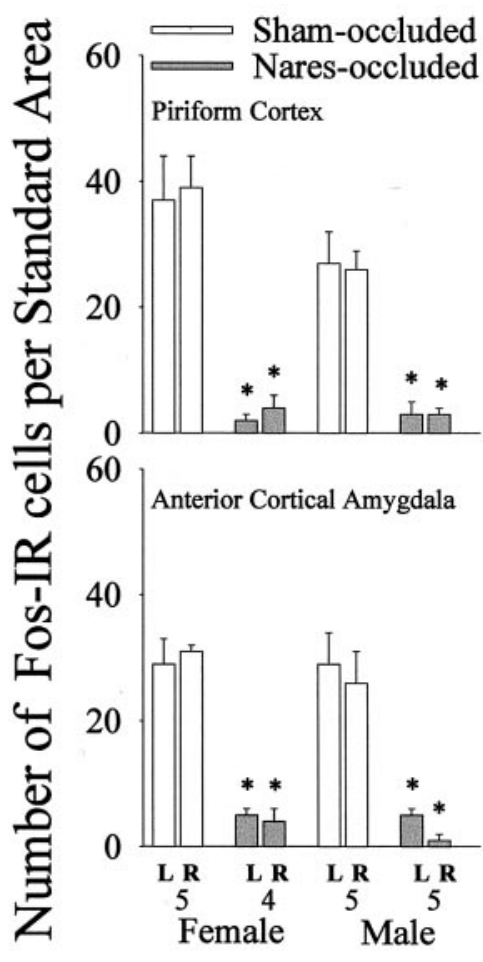

Figure 5. Effect of bilateral naris occlusion on neuronal Fos-immunoreactivity in the Pir and CoA of female and male ferrets killed at the end of this study after mating with an opposite-sex conspecific. The locations of the Fos counting regions (shaded areas) are shown for the Pir $(A)$ and CoA $(D)$. Photomicrographs of representative examples of Fos-IR neurons in these two brain regions are shown for a sham-occluded male $(B$, $E$ ) and a nares-occluded male $(C, F)$. BA, Basal-lateral amygdala; $F x$, fornix; $I C$, internal capsule; $L A$, lateral amygdala; $L O T$, lateral olfactory tract; $L v$, lateral ventricle; $M A$, medial amygdala; OT, optic tract. Right graphs, Quantitative Fos data for the Pir and CoA, left $(L)$ and right $(R)$ hemispheres. The number of subjects in each group is given beneath each pair of bars for the CoA results. ${ }^{*} p<0.01$, post hoc Student-Newman-Keuls comparisons with sham-occluded values for the same hemisphere. Data are expressed as mean \pm SEM.

sion material that was infused into the nasal cavity typically began to fill the nasal palatine canal without reaching the VNO duct. That neuronal Fos levels were equally low in the AOB cell layer of nares- and sham-occluded ferrets leaves open the possibility that nares occlusion disrupted heterosexual partner preference by blocking access of volatile odors to the VNO in addition to blocking their access to the MOE. This issue will only be resolved by directly assessing the effect of VNO destruction on ferrets' odor preferences and mate recognition.

Although the role of the MOE-MOB in the initial detection of socially relevant odors appears to be similar, the hypothalamic processing of these cues may differ in male and female ferrets (Kelliher et al., 1998). Thus in female subjects direct contact with soiled male bedding augmented neuronal Fos-IR in the medial POA and the ventrolateral hypothalamus, whereas contact with estrous female bedding only augmented Fos in the medial POA. Males showed no Fos responses to male or estrous female odors in either of these hypothalamic regions. Again, the absence of odor-induced hypothalamic Fos responses in males is not, by itself, evidence that odors failed to activate hypothalamic neurons in this sex. Instead, this finding points to a sex difference in the hypothalamic processing of main olfactory inputs, which, if understood, may clarify the neural basis of heterosexual mate recognition and preference in the two sexes.

A sex difference in nares-occluded ferrets' behavioral responses to conspecific cues was seen in preference tests given after subjects had received coital experience. We had expected heterosexual coital experience to increase the saliency of visual or auditory signals or both from opposite-sex ferrets so that nares-occluded subjects would prefer these as opposed to same-sex cues when Y maze testing was resumed. Instead, both male and female naresoccluded subjects continued to approach odor-only as well as odor plus visual plus auditory cues from stimulus males and females on an equal percentage of trails, and nares-occluded subjects actually had significantly longer approach latencies to both goal boxes than in tests given before coital experience. In the absence of data from nares-occluded ferrets whose preferences for these distal stimuli were simply retested without intervening coital experience, it is impossible to decide whether these longer approach latencies were the result of coital experience per se or, instead, reflected a reduction in the incentive value of these distal stimuli when presented repeatedly to these anosmic ferrets. It is notable, however, that when physical interaction with tethered stimulus ferrets was allowed after each trial, the latency of naresoccluded females to approach either stimulus animal was significantly shorter than in previous sessions in which only distal cues were provided. This effect was not seen in males, suggesting that olfactory perception is required to sustain all types of social motivation in this sex.

Our finding that female ferrets require volatile male odors for mate recognition corroborates previous studies in a variety of species showing that male odors can attract female conspecifics. Thus in invertebrates [e.g., moth (Rossler et al., 1999) and Drosophila (Higgie et al., 2000)], male pheromones play a central role in mate recognition by females. Likewise, among nonmammalian vertebrates, male pheromones that attract females have been 

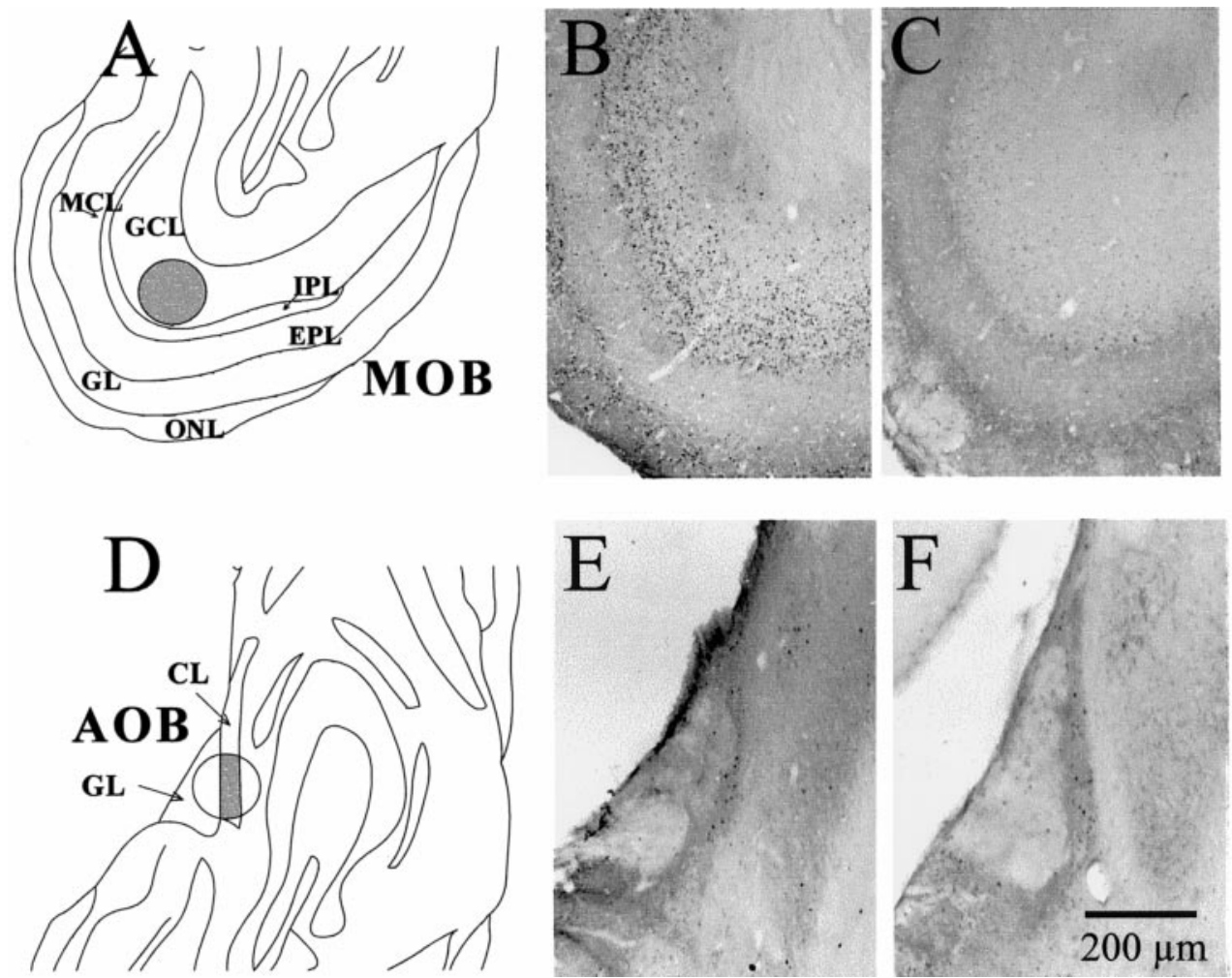

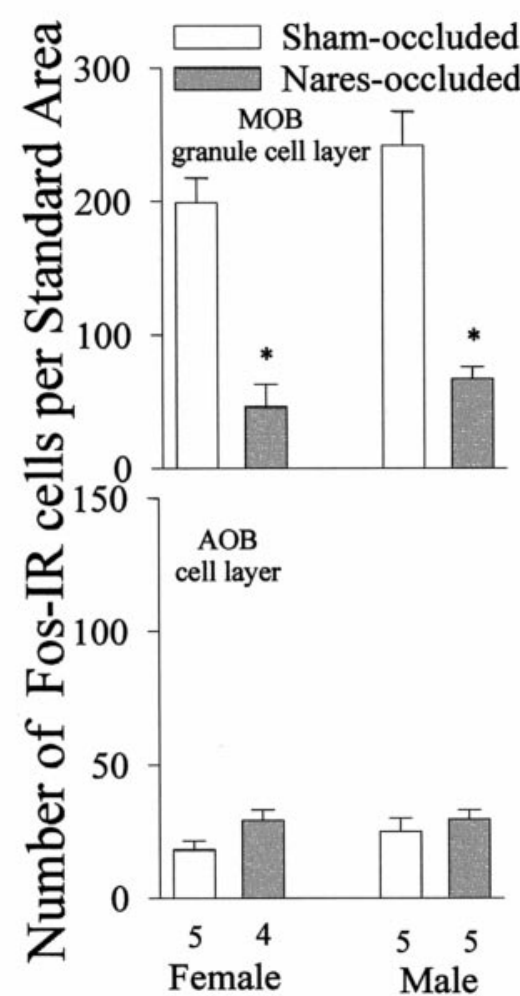

Figure 6. Effect of bilateral naris occlusion on neuronal Fos immunoreactivity in the granule cell layer $(G C L)$ of the MOB and in the cell layer $(C L)$ of the AOB of female and male ferrets killed at the end of this study after mating with an opposite-sex conspecific. The locations of the counting regions (shaded areas) are shown for the MOB $(A)$ and AOB $(D)$. Photomicrographs of representative examples of Fos-IR neurons in these two brain regions are shown for a sham-occluded male $(B, E)$ and a naris-occluded male $(C, F)$. EPL, External plexiform layer; $G L$, glomerular layer; $I P L$, internal plexiform layer; $M C L$, mitral cell layer; $O N L$, olfactory nerve layer. Right graphs, Quantitative Fos data for the MOB and AOB. The number of subjects in each group is given beneath the $A O B$ bars. ${ }^{*} p<0.01$, post hoc Student-Newman-Keuls comparisons with sham-occluded values of the same sex. Data are expressed as mean \pm SEM.

characterized biochemically in the newt (Kikuyama et al., 1995), salamander (Rollmann et al., 1999), and frog (Wabnitz et al., 1999; Pearl et al., 2000). In mice, two putative pheromones that are excreted in male urine, E,E, $\alpha$ - and $\mathrm{E}$ - $\beta$-farnesene, attract females (Jemiolo et al., 1991). In male pigs, androstenone, which is excreted in the saliva, attracts estrous sows (Signoret, 1970; Pearce and Hughes, 1987). Although the effect of peripheral anosmia has not been assessed, blocking the VNO ducts failed to alter sows' preference to approach androstenone, suggesting that the main olfactory system probably mediates its behavioral effect (Dorries et al., 1997). The present findings point to a similar odor detection mechanism in female ferrets.

Several previous studies (Powers et al., 1979; O'Connell and Meredith, 1984; Pfeiffer and Johnston, 1994) showed that chemical lesioning of the MOE in male hamsters reduced their interest in females' vaginal scents, although the ability of feminine visual and auditory cues to compensate for these deficits was not assessed. Michael and Keverne (1968) assessed the effects of peripheral anosmia on mate selection in two male rhesus monkeys. These males pressed a lever to gain access to an ovariectomized, estradiol-treated female, whereas they refused to press for ovariectomized females that received no steroids. After intranasal insertion of gauze plugs impregnated with bismuth-iodoformparaffin paste plus VNO nerve cuts, both males still refused to press the lever for access to these latter females even after they were given estradiol intravaginally. After removal of the nasal gauze plugs, however, both males pressed the lever for access to these females, presumably because they were attracted to vaginal odors emitted in response to estradiol. The fact that males continued pressing the lever for access to the original ovariectomized female that received systemic estradiol, even while nasal plugs were in place, suggests that visual or auditory cues or both were able to sustain their heterosexual partner preference. This outcome contrasts with the present study, in which the sight and sound of an estrous female ferret failed to compensate for the disruptive effect of peripheral anosmia on males' heterosexual partner preference. This species difference may reflect the greater cortical development in primates, leading to less dependence on any one sensory modality for mate recognition and sexual arousal (Dixson, 1998).

Bilateral naris occlusion failed to disrupt mating behavior in ferrets of either sex, suggesting that the MOE-MOB system is not essential for sexual arousal and coitus when animals are in close proximity. These results complement studies in the male hamster (Powers and Winans, 1973), dog (Hart and Haugen, 1971), and rhesus monkey (Michael and Keverne, 1968; Goldfoot et al., 1978) in which peripheral anosmia failed to disrupt sexual behavior. As already explained, we do not know whether the VNOAOB system is normally functional in ferrets, and we cannot rule out the possibility that nares occlusion disrupted ferrets' mate recognition by blocking the access of odors to the VNO in addition to the MOE. It is also possible, however, that the retention of coital behavior among nares-occluded ferrets reflected the presence of functional VNOs in these animals. Anogenital inves- 
tigation of stimulus animals was significantly reduced in naresoccluded ferrets of both sexes. Direct oral contact between ferrets was mainly limited to male subjects, all of which maintained a neck grip on an estrous stimulus female during tests of coital behavior. Thus nonvolatile odors from the skin of estrous females may have reached the male's VNO via the nasopalatine canal, thereby stimulating sexual arousal and coital performance. Nares-occluded females spent most of these tests receiving neck grips from stimulus males. Thus there was less opportunity for nonvolatile odors from the stimulus male to have gained access to the female's VNO. Again, this issue will only be resolved by directly assessing the effect of VNO destruction on ferrets' mating behavior.

That the ferret is able to use volatile body odors to identify potential mates at a distance is not surprising, nor is the finding that nares occlusion disrupted this ability unexpected. It is surprising, however, that anosmic ferrets of both sexes were unable to compensate for their olfactory deficit by using visual and auditory cues or the opportunity for direct physical interaction to guide them to opposite-sex partners. The VNO-AOB accessory olfactory system has received much attention in rodent studies of pheromonal communication (Keverne, 1999). The present study, although not ruling out a possible contribution of the $\mathrm{VNO}-\mathrm{AOB}$ system, establishes an obligatory role of the main olfactory system for heterosexual mate recognition in a carnivore.

\section{REFERENCES}

Adkins-Regan E (1988) Sex hormones and sexual orientation in animals. Psychobiology 16:335-347.

Bakker J, Kelliher KR, Baum MJ (2001) Mating induces gonadotropinreleasing hormone neuronal activation in anosmic female ferrets. Biol Reprod 64:1100-1105.

Baum MJ (1979) Differentiation of coital behavior in mammals: a comparative analysis. Neurosci Biobehav Rev 3:265-284.

Baum MJ, Carroll RS, Cherry JA, Tobet SA (1990a) Steroidal control of behavioral, neuroendocrine, and brain sexual differentiation: studies in a carnivore, the ferret. J Neuroendocrinol 2:401-418.

Baum MJ, Erskine MS, Kornberg E, Weaver CE (1990b) Prenatal and neonatal testosterone exposure interact to affect differentiation of sexual behavior and partner preference in female ferrets. Behav Neurosci 104:183-198.

Buchman CA, Doyle WJ, Swarts JD, Bluestone CD (1999) Effects of nasal obstruction on eustachian tube function and middle ear pressure. Acta Otolaryngol 119:351-355.

Chang YM, Kelliher KR, Baum MJ (2000) Steroidal modulation of scent investigation and marking behaviors in male and female ferrets. J Comp Psychol 114:401-407.

Clapperton BK, Minot EO, Crump DR (1988) An olfactory recognition system in the ferret, Mustela furo. Anim Behav 36:541-553.

Dixson AF (1998) Primate sexuality: comparative studies of the prosimians, monkeys, apes, and human beings. New York: Oxford UP.

Dorries KM, Adkins-Regan E, Halpern BP (1997) Sensitivity and behavioral responses to the pheromone androstenone are not mediated by the vomeronasal organ in domestic pigs. Brain Behav Evol 49:53-62.

Doty RL (1986) Odor-guided behavior in mammals. Experientia 42:257-271.

Edwards DA, Burge KG (1973) Olfactory control of the sexual behavior of male and female mice. Physiol Behav 11:867-872.

Goldfoot DA, Essock-Vitale SM, Asa CS, Thornton JE, Leshner AI (1978) Anosmia in male rhesus monkeys does not alter copulatory activity with cycling females. Science 199:1095-1096.
Hart BL, Haugen CM (1971) Scent marking and sexual behavior maintained in anosmic male dogs. Commun Behav Biol 6:131-135.

Higgie M, Chenoweth S, Blows MW (2000) Natural selection and the reinforcement of mate recognition. Science 290:519-521.

Jemiolo B, Xie TM, Novotny M (1991) Socio-sexual olfactory preference in female mice: attractiveness of synthetic chemosignals. Physiol Behav 50:1119-1122.

Kelliher KR, Chang YM, Wersinger SR, Baum MJ (1998) Sex difference and testosterone modulation of pheromone-induced neuronal fos in the ferret's main olfactory bulb and hypothalamus. Biol Reprod 59:1454-1463.

Kelliher KR, Baum MJ, Meredith M (2001) The ferret's vomeronasal organ and accessory olfactory bulb: effect of hormone manipulation in adult males and females. Anat Rec, in press.

Kelly JP, Wrynn AS, Leonard BE (1997) The olfactory bulbectomized rat as a model of depression: an update. Pharmacol Ther 74:299-316.

Kendrick KM, Atkins K, Hinton MR, Broad KD, Fabre-Nys C, Keverne EB (1995) Facial and vocal discrimination in sheep. Anim Behav 49:1665-1676.

Keverne EB (1999) The vomeronasal organ. Science 286:716-720.

Kikuyama S, Toyoda F, Ohmiya Y, Matsuda K, Tanaka S, Hayashi H (1995) Sodefrin: a female-attracting peptide pheromone in newt cloacal glands. Science 267:1643-1645.

Kindon HA, Baum MJ, Paredes RJ (1996) Medial preoptic/anterior hypothalamic lesions induce a female-typical profile of sexual partner preference in male ferrets. Horm Behav 30:514-527.

Michael RP, Keverne EB (1968) Pheromones in the communication of sexual status in primates. Nature 218:746-749.

Moors LM, Lavers RB (1981) Movements and home range of ferrets at Pukepuke Lagoon, New Zealand. N Z J Zool 8:413-423.

O'Connell RJ, Meredith M (1984) Effects of volatile and nonvolatile chemical signals on male sex behaviors mediated by the main and accessory olfactory systems. Behav Neurosci 98:1083-1093.

Paredes RG, Baum MJ (1995) Altered sexual partner preference in male ferrets given excitotoxic lesions of the preoptic area/anterior hypothalamus. J Neurosci 15:6619-6630.

Pearce GP, Hughes PE (1987) An investigation of the roles of boar component stimuli in the expression of proceptivity in the female. Appl Anim Behav 18:287-297.

Pearl CA, Cervantes M, Chan M, Ho U, Shoji R, Thomas EO (2000) Evidence for a mate-attracting chemosignal in the dwarf African frog. Horm Behav 38:67-74.

Pfeiffer CA, Johnston RE (1994) Hormonal and behavioral responses of male hamsters to females and female odors: roles of olfaction, the vomeronasal system, and sexual experience. Physiol Behav 55:129-138.

Powers JB, Winans SS (1973) Sexual behavior in peripherally anosmic male hamsters. Physiol Behav 10:361-368.

Powers JB, Fields RB, Winans SS (1979) Olfactory and vomeronasal system participation in male hamsters' attraction to female vaginal secretions. Physiol Behav 22:77-84.

Rollmann SM, Houck LD, Feldhoff RC (1999) Proteinaceous pheromone affecting female receptivity in a terrestrial salamander. Science 285:1907-1909.

Rossler W, Oland LA, Higgins MR, Hildebrand JG, Tolbert LP (1999) Development of a glia-rich axon-sorting zone in the olfactory pathway of the moth Manduca sexta. J Neurosci 19:9865-9877.

Signoret JP (1970) Reproductive behaviour of pigs. J Reprod Fertil [Suppl] 11:105-117.

Wabnitz PA, Bowie JH, Tyler MJ, Wallace JC, Smith BP (1999) Aquatic sex pheromone from a male tree frog. Nature 401:444-445.

Weiler E, Apfelbach R, Farbmen AL (1999) The vomeronasal organ of the male ferret. Chem Senses 24:127-139.

Wersinger SR, Baum MJ (1997) Sexually dimorphic processing of somatosensory and chemosensory inputs to forebrain luteinizing hormone-releasing hormone neurons in mated ferrets. Endocrinology 138:1121-1129.

Winer BJ, Brown DR, Michels KM (1991) Statistical principles in experimental design. New York: McGraw-Hill. 